\title{
Interprocedural Analysis with Lazy Propagation
}

\author{
Simon Holm Jensen ${ }^{1, \star}$, Anders Møller ${ }^{1, \star, \dagger}$, and Peter Thiemann ${ }^{2}$ \\ 1 Aarhus University, Denmark, \\ $\{$ simonh , amoeller\}@cs.au.dk \\ 2 Universität Freiburg, Germany, \\ thiemann@informatik. uni-freiburg.de
}

\begin{abstract}
We propose lazy propagation as a technique for flow- and context-sensitive interprocedural analysis of programs with objects and first-class functions where transfer functions may not be distributive. The technique is described formally as a systematic modification of a variant of the monotone framework and its theoretical properties are shown. It is implemented in a type analysis tool for JavaScript where it results in a significant improvement in performance.
\end{abstract}

\section{Introduction}

With the increasing use of object-oriented scripting languages, such as JavaScript, program analysis techniques are being developed as an aid to the programmers $[2,8-10,27,29]$. Although programs written in such languages are often relatively small compared to typical programs in other languages, their highly dynamic nature poses difficulties to static analysis. In particular, JavaScript programs involve complex interplays between first-class functions, objects with modifiable prototype chains, and implicit type coercions that all must be carefully modeled to ensure sufficient precision.

While developing a program analysis for JavaScript [15] aiming to statically infer type information we encountered the following challenge: How can we obtain a flow- and context-sensitive interprocedural dataflow analysis that accounts for mutable heap structures, supports objects and first-class functions, is amenable to non-distributive transfer functions, and is efficient and precise? Various directions can be considered. First, one may attempt to apply the classical monotone framework [18] as a whole-program analysis with an iterative fixpoint algorithm, where function call and return flow is treated as any other dataflow. This approach turns out to be unacceptable: the fixpoint algorithm requires too many iterations, and precision may suffer because spurious dataflow appears via interprocedurally unrealizable paths. Another approach is to apply the IFDS technique [23], which eliminates those problems. However, it is restricted to distributive analyses, which makes it inapplicable in our situation. A further consideration is the functional approach [26] which models each function in the program as a partial summary function that maps input dataflow

\footnotetext{
* Supported by The Danish Research Council for Technology and Production, grant no. 274-07-0488. ${ }^{\dagger}$ Corresponding author.
} 
facts to output dataflow facts and then uses this summary function whenever the function is called. However, with a dataflow lattice as large as in our case it becomes difficult to avoid reanalyzing each function a large number of times. Although there are numerous alternatives and variations of these approaches, we have been unable to find one in the literature that adequately addresses the challenge described above. Much effort has also been put into more specialized analyses, such as pointer analysis [11], however it is far from obvious how to generalize that work to our setting.

As an introductory example, consider this fragment of a JavaScript program:

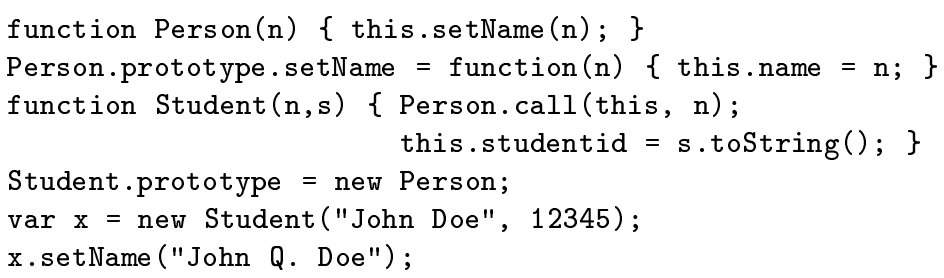

The code defines two "classes" with constructors Person and Student. Person has a method setName via its prototype object, and Student inherits setName and defines an additional field studentid. The call statement in Student invokes the super class constructor Person.

Analyzing the often intricate flow of control and data in such programs requires detailed modeling of points-to relations among objects and functions and of type coercion rules. TAJS is a whole-program analysis based on the monotone framework that follows this approach, and our first implementation is capable of analyzing complex properties of many JavaScript programs. However, our experiments have shown a considerable redundancy of computation during the analysis that causes simple functions to be analyzed a large number of times. If, for example, the setName method is called from other locations in the program, then the slightest change of any abstract state appearing at any call site of setName during the analysis would cause the method to be reanalyzed, even though the changes may be entirely irrelevant for that method. In this paper, we propose a technique for avoiding much of this redundancy while preserving, or even improving, the precision of the analysis. Although our main application is type analysis for JavaScript, we believe the technique is more generally applicable to analyses for object-oriented languages.

The main idea is to introduce a notion of "unknown" values for object fields that are not accessed within the current function. This prevents much irrelevant information from being propagated during the fixpoint computation. The analysis initially assumes that no fields are accessed when flow enters a function. When such an unknown value is read, a recovery operation is invoked to go back through the call graph and propagate the correct value. By avoiding to recover the same values repeatedly, the total amortized cost of recovery is never higher than that of the original analysis. With large abstract states, the mechanism makes a noticeable difference to the analysis performance. 
Lazy propagation should not be confused with demand-driven analysis [14]. The goal of the latter is to compute the results of an analysis only at specific program points thereby avoiding the effort to compute a global result. In contrast, lazy propagation computes a model of the state for each program point.

The contributions of this paper can be summarized as follows:

- We propose an ADT-based adaptation of the monotone framework to programming languages with mutable heap structures and first-class functions and exhibit some of its limitations regarding precision and performance.

- We describe a systematic modification of the framework that introduces lazy propagation. This novel technique propagates dataflow facts "by need" in an iterative fixpoint algorithm. We provide a formal description of the method to reason about its properties and to serve as a blueprint for an implementation.

- The lazy propagation technique is experimentally validated: It has been implemented into our type analysis for JavaScript, TAJS [15], resulting in a significant improvement in performance.

In the appendix we prove termination, relate lazy propagation with the basic framework - showing that precision does not decrease, and sketch a soundness proof of the analysis.

\section{A Basic Analysis Framework}

Our starting point is the classical monotone framework [18] tailored to programming languages with mutable heap structures and first-class functions. The mutable state consists of a heap of objects. Each object is a map from field names to values, and each value is either a reference to an object, a function, or some primitive value. Note that this section contains no new results, but it sets the stage for presenting our approach in Section 3.

\subsection{Analysis Instances}

Given a program $Q$, an instance of the monotone framework for an analysis of $Q$ is a tuple $\mathcal{A}=\left(F, N, L, P, C, n_{0}, c_{0}\right.$, Base, $\left.T\right)$ consisting of:

$F$ : the set of functions in $Q$;

$N$ : the set of primitive statements (also called nodes) in $Q$;

$L:$ a set of object labels in $Q$;

$P$ : a set of field names (also called properties) in $Q$;

$C$ : a set of abstract contexts, which are used for context sensitivity;

$n_{0} \in N$ and $c_{0} \in C$ : an initial statement and context describing the entry of $Q$;

Base: a base lattice for modeling primitive values, such as integers or booleans;

$T: C \times N \rightarrow$ AnalysisLattice $\rightarrow$ AnalysisLattice: a monotone transfer function for each primitive statement, where AnalysisLattice is a lattice derived from the above information as detailed in Section 2.2. 
Each of the sets must be finite and the Base lattice must have finite height. The primitive statements are organized into intraprocedural control flow graphs [19], and the set of object labels is typically determined by allocation-site abstraction $[5,16]$.

The notation fun $(n) \in F$ denotes the function that contains the statement $n \in N$, and entry $(f)$ and exit $(f)$ denote the unique entry statement and exit statement, respectively, of the function $f \in F$. For a function call statement $n \in N$, after $(n)$ denotes the statement being returned to after the call. A location is a pair $(c, n)$ of a context $c \in C$ and a statement $n \in N$.

\subsection{Derived Lattices}

An analysis instance gives rise to a collection of derived lattices. In the following, each function space is ordered pointwise and each powerset is ordered by inclusion. For a lattice $X$, the symbols $\perp_{X}$, $\sqsubseteq_{X}$, and $\sqcup_{X}$ denote the bottom element (representing the absence of information), the partial order, and the least upper bound operator (for merging information). We omit the $X$ subscript when it is clear from the context.

An abstract value is described by the lattice Value as a set of object labels, a set of functions, and an element from the base lattice:

$$
\text { Value }=\mathcal{P}(L) \times \mathcal{P}(F) \times \text { Base }
$$

An abstract object is a map from field names to abstract values:

$$
\mathrm{Obj}=P \rightarrow \text { Value }
$$

An abstract state is a map from object labels to abstract objects:

$$
\text { State }=L \rightarrow \text { Obj }
$$

Call graphs are described by this powerset lattice:

$$
\text { CallGraph }=\mathcal{P}(C \times N \times C \times F)
$$

In a call graph $g \in$ CallGraph, we interpret $\left(c_{1}, n_{1}, c_{2}, f_{2}\right) \in g$ as a potential function call from statement $n_{1}$ in context $c_{1}$ to function $f_{2}$ in context $c_{2}$.

Finally, an element of AnalysisLattice provides an abstract state for each context and primitive statement (in a forward analysis, the program point immediately before the statement), combined with a call graph:

$$
\text { AnalysisLattice }=(C \times N \rightarrow \text { State }) \times \text { CallGraph }
$$

In practice, an analysis may involve additional lattice components such as an abstract stack or extra information associated with each abstract object or field. We omit such components to simplify the presentation as they are irrelevant to the features that we focus on here. Our previous paper [15] describes the full lattices used in our type analysis for JavaScript. 




Fig. 1. The worklist algorithm. The worklist contains locations, i.e., pairs of a context and a statement. The operation $T_{a}(c, n)$ computes the transfer function for $(c, n)$ on the current analysis lattice element $a$ and updates $a$ accordingly. Additionally, it may add new entries to the worklist $W$. The transfer function for the initial location $\left(c_{0}, n_{0}\right)$ is responsible for creating the initial abstract state.

\subsection{Computing the Solution}

The solution to $\mathcal{A}$ is the least element $a \in$ AnalysisLattice that solves these constraints:

$$
\forall c \in C, n \in N: T(c, n)(a) \sqsubseteq a
$$

Computing the solution to the constraints involves fixpoint iteration of the transfer functions, which is typically implemented with a worklist algorithm as the one presented in Figure 1. The algorithm maintains a worklist $W \subseteq C \times N$ of locations where the abstract state has changed and thus the transfer function should be applied. Lattice elements representing functions, in particular $a \in$ AnalysisLattice, are generally considered as mutable and we use the notation $T_{a}(c, n)$ for the assignment $a:=T(c, n)(a)$. As a side effect, the call to $T_{a}(c, n)$ is responsible for adding entries to the worklist $W$, as explained in Section 2.4. This slightly unconventional approach to describing fixpoint iteration simplifies the presentation in the subsequent sections.

Note that the solution consists of both the computed call graph and an abstract state for each location. We do not construct the call graph in a preliminary phase because the presence of first-class functions implies that dataflow facts and call graph information are mutually dependent (as evident from the example program in Section 1).

This fixpoint algorithm leaves two implementation choices: the order in which entries are removed from the worklist $W$, which can greatly affect the number of iterations needed to reach the fixpoint, and the representation of lattice elements, which can affect both time and memory usage. These choices are, however, not the focus of the present paper (see, e.g. $[3,13,17,19,28]$ ).

\subsection{An Abstract Data Type for Transfer Functions}

To precisely explain our modifications of the framework in the subsequent sections, we treat AnalysisLattice as an imperative ADT (abstract data type) [20] with the following operations: 
- getfield : $C \times N \times L \times P \rightarrow$ Value

- getcallgraph $:() \rightarrow$ CallGraph

- getstate : $C \times N \rightarrow$ State

- propagate $: C \times N \times$ State $\rightarrow()$

- funentry : $C \times N \times C \times F \times$ State $\rightarrow()$

- funexit : $C \times N \times C \times F \times$ State $\rightarrow()$

We let $a \in$ AnalysisLattice denote the current, mutable analysis lattice element. The transfer functions can only access $a$ through these operations.

The operation getfield $(c, n, \ell, p)$ returns the abstract value of the field $p$ in the abstract object $\ell$ at the entry of the primitive statement $n$ in context $c$. In the basic framework, getfield performs a simple lookup, without any side effects on the analysis lattice element:

a.getfield $(c \in C, n \in N, \ell \in L, p \in P)$ :

return $u(\ell)(p)$ where $\left(m,{ }_{-}\right)=a$ and $u=m(c, n)$

The getcallgraph operation selects the call graph component of the analysis lattice element:

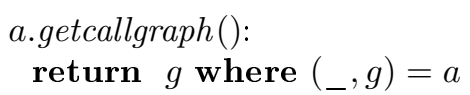

Transfer functions typically use the getcallgraph operation in combination with the funexit operation explained below. Moreover, the getcallgraph operation plays a role in the extended framework presented in Section 3.

The getstate operation returns the abstract state at a given location:

$$
\begin{aligned}
& \text { a.getstate }(c \in C, n \in N): \\
& \quad \text { return } m(c, n) \text { where }\left(m,{ }_{-}\right)=a
\end{aligned}
$$

The transfer functions must not read the field values from the returned abstract state (for that, the getfield operation is to be used). They may construct parameters to the operations propagate, funentry, and funexit by updating a copy of the returned abstract state.

The transfer functions must use the operation propagate $(c, n, s)$ to pass information from one location to another within the same function (excluding recursive function calls). As a side effect, propagate adds the location $(c, n)$ to the worklist $W$ if its abstract state has changed. In the basic framework, propagate is defined as follows:

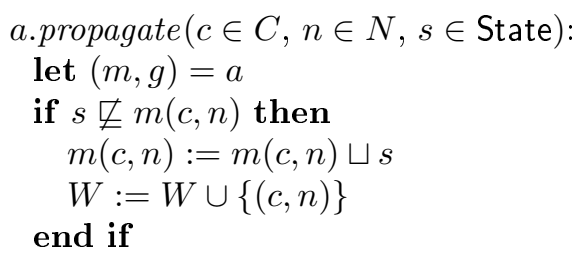

The operation funentry $\left(c_{1}, n_{1}, c_{2}, f_{2}, s\right)$ models function calls in a forward analysis. It modifies the analysis lattice element $a$ to reflect the possibility of a 
function call from a statement $n_{1}$ in context $c_{1}$ to a function entry statement entry $\left(f_{2}\right)$ in context $c_{2}$ where $s$ is the abstract state after parameter passing. (With languages where parameters are passed via the stack, which we ignore here, the lattice is augmented accordingly.) In the basic framework, funentry adds the call edge from $\left(c_{1}, n_{1}\right)$ to $\left(c_{2}, f_{2}\right)$ and propagates $s$ into the abstract state at the function entry statement $\operatorname{entry}\left(f_{2}\right)$ in context $c_{2}$ :

$$
\begin{aligned}
& \text { a.funentry }\left(c_{1} \in C, n_{1} \in N, c_{2} \in C, f_{2} \in F, s \in \text { State }\right) \text { : } \\
& g:=g \cup\left\{\left(c_{1}, n_{1}, c_{2}, f_{2}\right)\right\} \text { where }\left({ }_{-}, g\right)=a \\
& \text { a.propagate }\left(c_{2}, \operatorname{entry}\left(f_{2}\right), s\right) \\
& \text { a.funexit }\left(c_{1}, n_{1}, c_{2}, f_{2}, m\left(c_{2}, \operatorname{exit}\left(f_{2}\right)\right)\right)
\end{aligned}
$$

Adding a new call edge also triggers a call to funexit to establish dataflow from the function exit to the successor of the new call site.

The operation funexit $\left(c_{1}, n_{1}, c_{2}, f_{2}, s\right)$ is used for modeling function returns. It modifies the analysis lattice element to reflect the dataflow of $s$ from the exit of a function $f_{2}$ in callee context $c_{2}$ to the successor of the call statement $n_{1}$ with caller context $c_{1}$. The basic framework does so by propagating $s$ into the abstract state at the latter location:

$$
\begin{aligned}
& \text { a.funexit }\left(c_{1} \in C, n_{1} \in N, c_{2} \in C, f_{2} \in F, s \in \text { State }\right) \text { : } \\
& \quad \text { a.propagate }\left(c_{1}, \operatorname{after}\left(n_{1}\right), s\right)
\end{aligned}
$$

The parameters $c_{2}$ and $f_{2}$ are not used in the basic framework; they will be used in Section 3. The transfer functions obtain the connections between callers and callees via the getcallgraph operation explained earlier. If using an augmented lattice where the call stack is also modeled, that component would naturally be handled differently by funexit simply by copying it from the call location $\left(c_{1}, n_{1}\right)$, essentially as local variables are treated in, for example, IFDS [23].

This basic framework is sufficiently general as a foundation for many analyses for object-oriented programming languages, such as Java or C\#, as well as for object-based scripting languages like JavaScript as explained in Section 4. At the same time, it is sufficiently simple to allow us to precisely demonstrate the problems we attack and our solution in the following sections.

\subsection{Problems with the Basic Analysis Framework}

The first implementation of TAJS, our program analysis for JavaScript, is based on the basic analysis framework. Our initial experiments showed, perhaps not surprisingly, that many simple functions in our benchmark programs were analyzed over and over again (even for the same calling contexts) until the fixpoint was reached.

For example, a function in the richards.js benchmark from the V8 collection was analyzed 18 times when new dataflow appeared at the function entry:

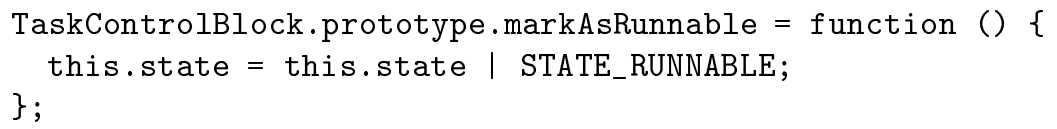


Most of the time, the new dataflow had nothing to do with the this object or the STATE_RUNNABLE variable. Although this particular function body is very short, it still takes time and space to analyze it and similar situations were observed for more complex functions and in other benchmark programs.

In addition to this abundant redundancy, we observed - again not surprisingly - a significant amount of spurious dataflow resulting from interprocedurally invalid paths. For example, if the function above is called from two different locations, with the same calling context, their entire heap structures (that is, the State component in the lattice) become joined, thereby losing precision.

Another issue we noticed was time and space required for propagating the initial state, which consists of 161 objects in the case of JavaScript. These objects are mutable and the analysis must account for changes made to them by the program. Since the analysis is both flow- and context-sensitive, a typical element of AnalysisLattice carries a lot of information even for small programs.

Our first version of TAJS applied two techniques to address these issues: (1) Lattice elements were represented in memory using copy-on-write to make their constituents shared between different locations until modified. (2) The lattice was extended to incorporate a simple effect analysis called maybe-modified: For each object field, the analysis would keep track of whether the field might have been modified since entering the current function. At function exit, field values that were definitely not modified by the function would be replaced by the value from the call site. As a consequence, the flow of unmodified fields was not affected by function calls. Although these two techniques are quite effective, the lazy propagation approach that we introduce in the next section often supersedes the maybe-modified technique and renders copy-on-write essentially superfluous. In Section 4 we experimentally compare lazy propagation with both the basic framework and the basic framework extended with the copy-on-write and maybemodified techniques.

\section{Extending the Framework with Lazy Propagation}

To remedy the shortcomings of the basic framework, we propose an extension that can help reducing the observed redundancy and the amount of information being propagated by the transfer functions. The key idea is to ensure that the fixpoint solver propagates information "by need". The extension consists of a systematic modification of the ADT representing the analysis lattice. This modification implicitly changes the behavior of the transfer functions without touching their implementation.

\subsection{Modifications of the Analysis Lattice}

In short, we modify the analysis lattice as follows:

1. We introduce an additional abstract value, unknown. Intuitively, a field $p$ of an object has this value in an abstract state associated with some location in 
a function $f$ if the value of $p$ is not known to be needed (that is, referenced) in $f$ or in a function called from $f$.

2. Each call edge is augmented with an abstract state that captures the data flow along the edge after parameter passing, such that this information is readily available when resolving unknown field values.

3. A special abstract state, none, is added, for describing absent call edges and locations that may be unreachable from the program entry.

More formally, we modify three of the sub-lattices as follows:

$$
\begin{gathered}
\text { Obj }=P \rightarrow\left(\text { Value } \downarrow_{\text {unknown }}\right) \\
\text { CallGraph }=C \times N \times C \times F \rightarrow\left(\text { State } \downarrow_{\text {none }}\right) \\
\text { AnalysisLattice }=\left(C \times N \rightarrow\left(\text { State } \downarrow_{\text {none }}\right)\right) \times \text { CallGraph }
\end{gathered}
$$

Here, $X \downarrow_{\text {y }}$ means the lattice $X$ lifted over a new bottom element y. In a call graph $g \in$ CallGraph in the original lattice, the presence of an edge $\left(c_{1}, n_{1}, c_{2}, f_{2}\right) \in g$ is modeled by $g^{\prime}\left(c_{1}, n_{1}, c_{2}, f_{2}\right) \neq$ none for the corresponding call graph $g^{\prime}$ in the modified lattice. Notice that $\perp_{\text {State }}$ is now the function that maps all object labels and field names to unknown, which is different from the element none.

\subsection{Modifications of the Abstract Data Type Operations}

Before we describe the systematic modifications of the ADT operations we motivate the need for an auxiliary operation, recover, on the ADT:

$$
\text { recover : } C \times N \times L \times P \rightarrow \text { Value }
$$

Suppose that, during the fixpoint iteration, a transfer function $T_{a}(c, n)$ invokes a.getfield $(c, n, \ell, p)$ with the result unknown. This result indicates the situation that the field $p$ of an abstract object $\ell$ is referenced at the location $(c, n)$, but the field value has not yet been propagated to this location due to the lazy propagation. The recover operation can then compute the proper field value by performing a specialized fixpoint computation to propagate just that field value to $(c, n)$. We explain in Section 3.3 how recover is defined.

The getfield operation is modified such that it invokes recover if the desired field value is unknown, as shown in Figure 2. The modification may break monotonicity of the transfer functions, however, as we argue in Appendix A, the analysis still produces the correct result.

Similarly, the propagate operation needs to be modified to account for the lattice element none and for the situation where unknown is joined with an ordinary element. The latter is accomplished by using recover whenever this situation occurs. The resulting operation propagate' is shown in Figure 3.

We then modify funentry $\left(c_{1}, n_{1}, c_{2}, f_{2}, s\right)$ such that the abstract state $s$ is propagated "lazily" into the abstract state at the primitive statement $\operatorname{entry}\left(f_{2}\right)$ in context $c_{2}$. Here, laziness means that every field value that, according to $a$, is not referenced within the function $f_{2}$ in context $c_{2}$ gets replaced by unknown in the abstract state. Additionally, the modified operation records the abstract state at the call edge as required in the modified CallGraph lattice. The resulting 


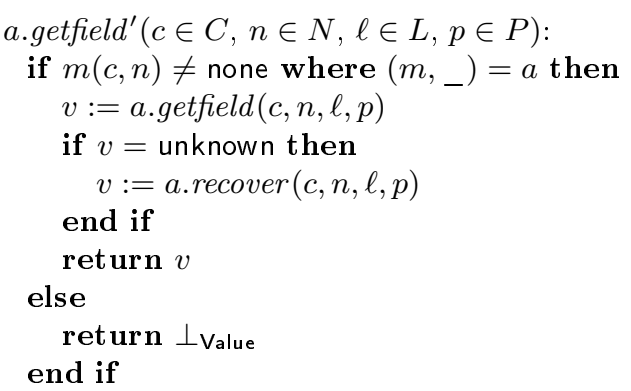

Fig. 2. Algorithm for getfield $(c, n, \ell, p)$. This modified version of getfield invokes recover in case the desired field value is unknown. If the state is none according to $a$, the operation simply returns $\perp_{\text {Value }}$.

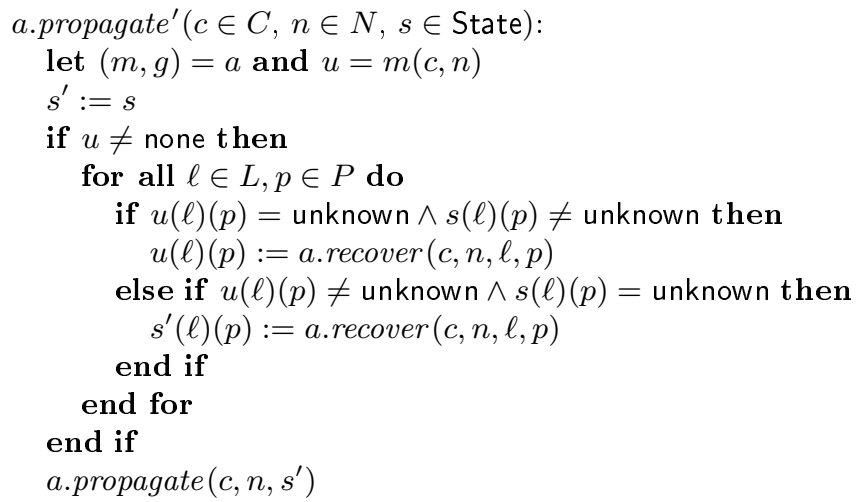

Fig. 3. Algorithm for propagate $(c, n, s)$. This modified version of propagate takes into account that field values may be unknown in both $a$ and $s$. Specifically, it uses recover to ensure that the invocation of propagate in the last line never computes the least upper bound of unknown and an ordinary field value. The treatment of unknown values in $s$ assumes that $s$ is recoverable with respect to the current location $(c, n)$. If the abstract state at $(c, n)$ is none (the least element), then that gets updated to $s$.

operation funentry' is defined in Figure 4. (Without loss of generality, we assume that the statement at $\operatorname{exit}\left(f_{2}\right)$ returns to the caller without modifying the state.) As consequence of the modification, unknown field values get introduced into the abstract states at function entries.

The funexit operation is modified such that every unknown field value appearing in the abstract state being returned is replaced by the corresponding field value from the call edge, as shown in Figure 5. In JavaScript, entering a function body at a functions call affects the heap, which is the reason for using the state from the call edge rather than the state from the call statement. If we extended the lattice to also model the call stack, then that component would naturally be recovered from the call statement rather than the call edge.

Figure 6 illustrates the dataflow at function entries and exits as modeled by the funexit' and funentry' operations. The two nodes $n_{1}$ and $n_{2}$ represent 




Fig. 4. Algorithm for funentry' $\left(c_{1}, n_{1}, c_{2}, f_{2}, s\right)$. This modified version of funentry "lazily" propagates $s$ into the abstract state at $\operatorname{entry}\left(f_{2}\right)$ in context $c_{2}$. The abstract state $s^{\prime}$ is unknown for all fields that have not yet been referenced by the function being called according to $u$ (recall that $\perp_{\text {State }}$ maps all fields to unknown).

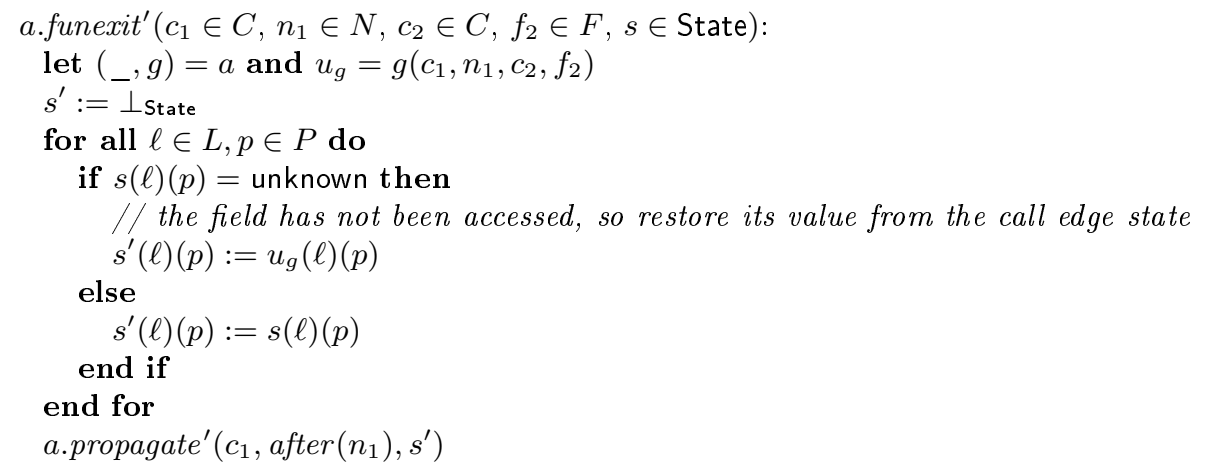

Fig. 5. Algorithm for funexit' $\left(c_{1}, n_{1}, c_{2}, f_{2}, s\right)$. This modified version of funexit restores field values that have not been accessed within the function being called, using the value from before the call. It then propagates the resulting state as in the original operation.

function call statements that invoke the function $f$. Assume that the value of the field $p$ in the abstract object $\ell$, denoted $\ell . p$, is $v_{1}$ at $n_{1}$ and $v_{2}$ at $n_{2}$ where $v_{1}, v_{2} \in$ Value. When dataflow first arrives at entry $(f)$ the funentry' operation sets $\ell . p$ to unknown. Assuming that $f$ does not access $\ell . p$ it remains unknown throughout $f$, so funexit' can safely restore the original value $v_{1}$ by merging the state from exit $(f)$ with $u_{g 1}$ (the state recorded at the call edge) at after $\left(n_{1}\right)$. 


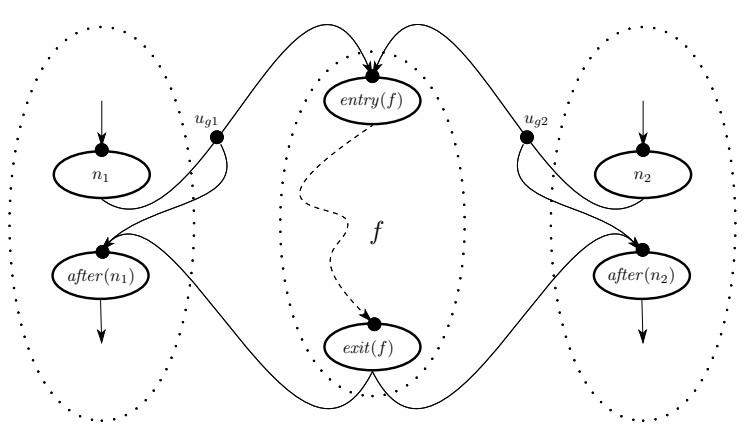

Fig. 6. A function $f$ being called from two different statements, $n_{1}$ and $n_{2}$ appearing in other functions (for simplicity, all with the same context $c$ ). The edges indicate dataflow, and each bullet corresponds to an element of State with $u_{g 1}=g\left(c, n_{1}, c, f\right)$ and $u_{g 2}=g\left(c, n_{2}, c, f\right)$ where $g \in$ CallGraph.

Similarly for the other call site, the value $v_{2}$ will be restored at after $\left(n_{2}\right)$. Thus, the dataflow for non-referenced fields respects the interprocedurally valid paths. This is in contrast to the basic framework where the value of $\ell . p$ would be $v_{1} \sqcup v_{2}$ at both after $\left(n_{1}\right)$ and after $\left(n_{2}\right)$. Thereby, the modification of funexit may - perhaps surprisingly - cause the resulting analysis solution to be more precise than in the basic framework. If a statement in $f$ writes a value $v^{\prime}$ to $\ell$.p it will no longer be unknown, so $v^{\prime}$ will propagate to both after $\left(n_{1}\right)$ and after $\left(n_{2}\right)$. If the transfer function of a statement in $f$ invokes getfield ${ }^{\prime}$ to obtain the value of $\ell . p$ while it is unknown, it will be recovered by considering the call edges into $f$, as explained in Section 3.3.

The getstate operation is not modified. A transfer function cannot notice the fact that the returned State elements may contain unknown field values, because it is not permitted to read a field value through such a state.

Finally, the getcallgraph operation requires a minor modification to ensure that its output has the same type although the underlying lattice has changed:

a.getcallgraph ${ }^{\prime}()$ :

return $\left\{\left(c_{1}, n_{1}, c_{2}, f_{2}\right) \mid g\left(c_{1}, n_{1}, c_{2}, f_{2}\right) \neq\right.$ none $\}$ where $\left(_{-}, g\right)=a$

To demonstrate how the lazy propagation framework manages to avoid certain redundant computations, consider again the markAsRunnable function in Section 2.5. Suppose that the analysis first encounters a call to this function with some abstract state $s$. This call triggers the analysis of the function body, which accesses only a few object fields within $s$. The abstract state at the entry location of the function is unknown for all other fields. If new flow subsequently arrives via a call to the function with another abstract state $s^{\prime}$ where $s \sqsubseteq s^{\prime}$, the introduction of unknown values ensures that the function body is only reanalyzed if $s^{\prime}$ differs from $s$ at the few relevant fields that are not unknown.

\subsection{Recovering Unknown Field Values}

We now turn to the definition of the auxiliary operation recover. It gets invoked by getfield' and propagate' whenever an unknown element needs to be replaced 
by a proper field value. The operation returns the desired field value but also, as a side effect, modifies the relevant abstract states for function entry locations in $a$.

The key observation for defining recover $(c, n, \ell, p)$ where $c \in C, n \in N, \ell \in L$, and $p \in P$ is that unknown is only introduced in funentry' and that each call edge - very conveniently - records the abstract state just before the ordinary field value is changed into unknown. Thus, the operation needs to go back through the call graph and recover the missing information. However, it only needs to modify the abstract states that belong to function entry statements.

Recovery is a two phase process. The first phase constructs a directed multirooted graph $G$ the nodes of which are a subset of $C \times F$. It is constructed from the call graph in a backward manner starting from $(c, n)$ as the smallest graph satisfying the following two constraints, where $(m, g)=a$ :

- The graph $G$ contains the node $(c, f u n(n)) .^{3}$

- For each node $\left(c_{2}, f_{2}\right)$ in $G$ and for each $\left(c_{1}, n_{1}\right)$ where $g\left(c_{1}, n_{1}, c_{2}, f_{2}\right) \neq$ none:

- If $u_{g}(\ell)(p)=$ unknown $\wedge u_{1}(\ell)(p)=$ unknown where $u_{g}=g\left(c_{1}, n_{1}, c_{2}, f_{2}\right)$ and $u_{1}=m\left(c_{1}, \operatorname{entry}\left(f u n\left(n_{1}\right)\right)\right)$ then $G$ contains the node $\left(c_{1}\right.$, fun $\left.\left(n_{1}\right)\right)$ with an edge to $\left(c_{2}, f_{2}\right)$,

- otherwise, $\left(c_{2}, f_{2}\right)$ is a root of $G$.

The resulting graph is essentially a subgraph of the call graph. A node in $G$ is a root if at least one of the incoming call graph edges of the corresponding function contributes with a non-unknown value. Notice that root nodes may have incoming edges in $G$.

The second phase is a fixpoint computation over $G$ :



\footnotetext{
${ }^{3}$ This constraint has been corrected after the SAS 2010 paper was published.
} 




This phase recovers the abstract value at the roots of $G$ and then propagates the value through the nodes of $G$ until a fixpoint is reached. Although recover modifies abstract states in this phase, it does not modify the worklist, an issue which we return to in Appendix A.3. After this phase, we have $u(\ell)(p) \neq$ unknown where $u=m\left(c^{\prime}\right.$, entry $\left.\left(f^{\prime}\right)\right)$ for each node $\left(c^{\prime}, f^{\prime}\right)$ in $G$. (Notice that the side effects on $a$ only concern abstract states at function entry statements.) In particular, this holds for $(c, f u n(n))$, so when recover $(c, n, \ell, p)$ has completed the two phases, it returns the desired value $u(\ell)(p)$ where $u=m(c, \operatorname{entry}(f u n(n)))$.

Notice that the graph $G$ is empty if $u(\ell)(p) \neq$ unknown where $u=m(c$, entry $(f u n(n))$ ) (see the first of the two constraints defining $G$ ). In this case, the desired field has already been recovered, the second phase is effectively skipped, and $u(\ell)(p)$ is returned immediately.

Figure 7 illustrates an example of interprocedural dataflow among four functions. (This example ignores dataflow for function returns and assumes a fixed calling context c.) The statements write $_{1}$ and write $_{2}$ write to a field $\ell . p$, and read reads from it. Assume that the analysis discovers all the call edges before visiting read. In that case, $\ell . p$ will have the value unknown when entering $f_{2}$ and $f_{3}$, which will propagate to $f_{4}$. The transfer function for read will then invoke getfield', which in turn invokes recover. The graph $G$ will be constructed with three nodes: $\left(c, f_{2}\right),\left(c, f_{3}\right)$, and $\left(c, f_{4}\right)$ where $\left(c, f_{2}\right)$ and $\left(c, f_{3}\right)$ are roots and have edges to $\left(c, f_{4}\right)$. The second phase of recover will replace the unknown value of $\ell . p$ at entry $\left(f_{2}\right)$ and entry $\left(f_{2}\right)$ by its proper value stored at the call edges and then propagate that value to entry $\left(f_{3}\right)$ and finally return it to getfield'. Notice that the value of $\ell . p$ at, for example, the call edges, remains unknown. However, if dataflow subsequently arrives via transfer functions of other statements, those unknown values may be replaced by ordinary values. Finally, note that this simple example does not require fixpoint iteration within recover, however that becomes necessary when call graphs contain cycles (resulting from programs with recursive function calls).

The modifications only concern the AnalysisLattice ADT, in terms of which all transfer functions of an analysis are defined. The transfer functions themselves are not changed. Although invocations of recover involve traversals of parts of the call graph, the main worklist algorithm (Figure 1) requires no modifications.

\section{Implementation and Experiments}

To examine the impact of lazy propagation on analysis performance, we extended the Java implementation of TAJS, our type analyzer for JavaScript [15], 


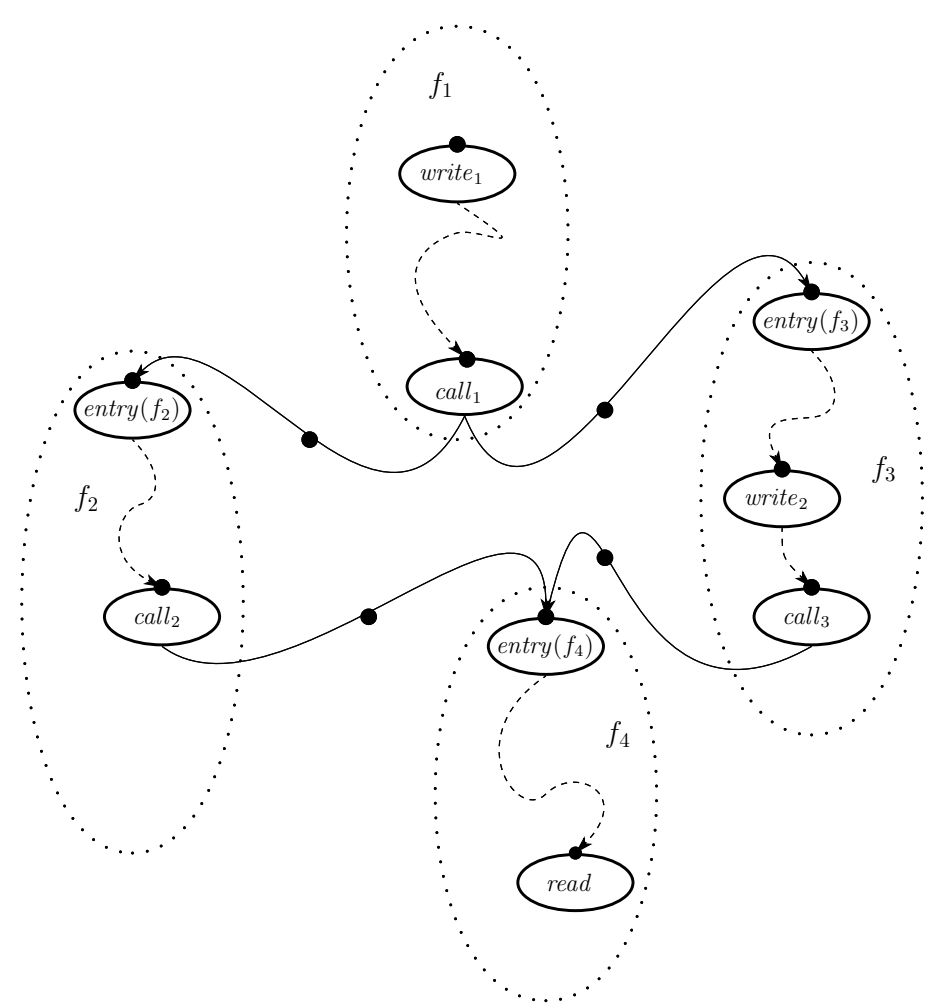

Fig. 7. Fragments of four functions, $f_{1} \ldots f_{4}$. As in Figure 6, edges indicate dataflow and bullets correspond to elements of State. The statements write $_{1}$ and write $_{2}$ write to a field $\ell . p$, and read reads from it. The recover operation applied to the read statement and $\ell . p$ will ensure that values written at write $_{1}$ and write $_{2}$ will be read at the read statements, despite the possible presence of unknown values.

by systematically applying the modifications described in Section 3. As usual in dataflow analysis, primitive statements are grouped into basic blocks. The implementation focuses on the JavaScript language itself and the built-in library, but presently excludes the DOM API, so we use the most complex benchmarks from the $\mathrm{V}^{4}$ and SunSpider ${ }^{5}$ benchmark collections for the experiments.

Descriptions of other aspects of TAJS not directly related to lazy propagation may be found in the TAJS paper [15]. These include the use of recency abstraction [4], which complicates the implementation, but does not change the properties of the lazy propagation technique.

We compare three versions of the analysis: basic corresponds to the basic framework described in Section 2; basic + extends the basic version with the copyon-write and maybe-modified techniques discussed in Section 2.5, which is the version used in [15]; and lazy is the new implementation using lazy propagation (without the other extensions from the basic+ version).

\footnotetext{
${ }^{4}$ http://v8.googlecode.com/svn/data/benchmarks/v1/

${ }^{5}$ http://www2 . webkit.org/perf/sunspider-0.9/sunspider.html
} 


\begin{tabular}{|l|r|r|r|r|r|r|r|r|r|r|r|}
\hline & & & \multicolumn{3}{|c|}{ Iterations } & \multicolumn{3}{|c|}{ Time (seconds) } & \multicolumn{3}{|c|}{ Memory (MB) } \\
\cline { 4 - 10 } & LOC & Blocks & basic & basic+ & lazy & basic & basic+ & lazy & basic & basic + & lazy \\
\hline richards.js & 529 & 478 & 2663 & 2782 & 1399 & 5.6 & 4.6 & 3.8 & 11.05 & 6.4 & 3.7 \\
benchpress.js & 463 & 710 & 18060 & 12581 & 5097 & 33.2 & 13.4 & 5.4 & 42.02 & 24.0 & 7.8 \\
delta-blue.js & 853 & 1054 & $\infty$ & $\infty$ & 63611 & $\infty$ & $\infty$ & 136.7 & $\infty$ & $\infty$ & 140.5 \\
cryptobench.js & 1736 & 2857 & $\infty$ & 43848 & 17213 & $\infty$ & 99.4 & 22.1 & $\infty$ & 127.9 & 42.8 \\
\hline 3d-cube.js & 342 & 545 & 7116 & 4147 & 2009 & 14.1 & 5.3 & 4.0 & 18.4 & 10.6 & 6.2 \\
3d-raytrace.js & 446 & 575 & $\infty$ & 30323 & 6749 & $\infty$ & 24.8 & 8.2 & $\infty$ & 16.7 & 10.1 \\
crypto-md5.js & 296 & 392 & 5358 & 1004 & 646 & 4.5 & 2.0 & 1.8 & 6.1 & 3.6 & 2.7 \\
access-nbody.js & 179 & 149 & 551 & 523 & 317 & 1.8 & 1.3 & 1.0 & 3.2 & 1.7 & 0.9 \\
\hline
\end{tabular}

Table 1. Performance benchmark results.

Table 1 shows for each program, the number of lines of code, the number of basic blocks, the number of fixpoint iterations for the worklist algorithm (Figure 1), analysis time (in seconds, running on a $3.2 \mathrm{GHz} \mathrm{PC}$ ), and memory consumption. We use $\infty$ to denote runs that require more than 512MB of memory.

We focus on the time and space requirements for these experiments. On our benchmark programs, the precision improvement is insignificant with respect to the number of potential type related bugs, which is the precision measure we have used in our previous work.

The experiments demonstrate that although the copy-on-write and maybemodified techniques have a significant positive effect on the resource requirements, lazy propagation leads to even better results. The results for richards . js are a bit unusual as it takes more iterations in basic + than in basic, however the fixpoint is more precise in basic + .

The benchmark results demonstrate that lazy propagation results in a significant reduction of analysis time without sacrificing precision. Memory consumption is reduced by propagating less information during the fixpoint computation and fixpoints are reached in fewer iterations by eliminating a cause of redundant computation observed in the basic framework.

\section{Related Work}

Recently, JavaScript and other scripting languages have come into the focus of research on static program analysis, partly because of their challenging dynamic nature. These works range from analysis for security vulnerabilities $[9,29]$ to static type inference $[1,8,15,27]$. We concentrate on the latter category, aiming to develop program analyses that can compensate for the lack of static type checking in these languages. The interplay of language features of JavaScript, including first-class functions, objects with modifiable prototype chains, and implicit type coercions, makes analysis a demanding task.

The IFDS framework by Reps, Horwitz, and Sagiv [23] is a powerful and widely used approach for obtaining precise interprocedural analyses. It requires the underlying lattice to be a powerset and the transfer functions to be distributive. Unfortunately, these requirements are not met by our type analysis problem for dynamic object-oriented scripting languages. The more general IDE framework also requires distributive transfer functions [25]. A connection to our approach is that fields that are marked as unknown at function exits, and hence 
have not been referenced within the function, are recovered from the call site in the same way local variables are treated in IFDS.

Sharir and Pnueli's functional approach to interprocedural analysis can be phrased both with symbolic representations and in an iterative style [26], where the latter is closer to our approach. With the complex lattices and transfer functions that appear to be necessary in analyses for object-oriented scripting languages, symbolic representations are difficult to work with, so TAJS uses the iterative style and a relatively direct representation of lattice elements. Furthermore, the functional approach is expensive if the analysis lattice is large.

Our analysis framework encompasses a general notion of context sensitivity through the $C$ component of the analysis instances. Different instantiations of $C$ lead to different kinds of context sensitivity, including variations of the call-string approach [26], which may also affect the quality of interprocedural analysis. We leave the choice of $C$ open here; TAJS currently uses a heuristic that distinguishes call sites that have different values of this.

The use of unknown field values is related to the maybe-modified technique that we used in the first version of TAJS [15]: a field whose value is unknown is definitely not modified. Both ideas can be viewed as instances of side effect analysis. Unlike, for example, the side effect analysis by Landi et al. [24] our analysis computes the call graph on-the-fly and we exploit the information that certain fields are found to be non-referenced for obtaining the lazy propagation mechanism. Via this connection to side effect analysis, one may also view the unknown field values as establishing a frame condition as in separation logic [21].

Combining call graph construction with other analyses is common in pointer alias analysis with function pointers, for example in the work of Burke et al. [12]. That paper also describes an approach called deferred evaluation for increasing analysis efficiency, which is specialized to flow insensitive alias analysis.

Lazy propagation is related to lazy evaluation (e.g., [22]) as it produces values passed to functions on demand, but there are some differences. Lazy propagation does not defer evaluation as such, but just the propagation of the values; it applies not just to the parameters but to the entire state; and it restricts laziness to data structures (values of fields).

Lazy propagation is different from demand-driven analysis [14]. Both approaches defer computation, but demand-driven analysis only computes results for selected hot spots, whereas our goal is a whole-program analysis that infers information for all program points. Other techniques for reducing the amount of redundant computation in fixpoint solvers is difference propagation [7] and use of interprocedural def-use chains [28]. It might be possible to combine those techniques with lazy propagation, although they are difficult to apply to the complex transfer functions that we have in type analysis for JavaScript.

\section{Conclusion}

We have presented lazy propagation as a technique for improving the performance of interprocedural analysis in situations where existing methods, such as IFDS or the functional approach, do not apply. The technique is described by a 
systematic modification of a basic iterative framework. Through an implementation that performs type analysis for JavaScript we have demonstrated that it can significantly reduce the memory usage and the number of fixpoint iterations without sacrificing analysis precision. The result is a step toward sound, precise, and fast static analysis for object-oriented languages in general and scripting languages in particular.

Acknowledgments The authors thank Stephen Fink, Michael Hind, and Thomas Reps for their inspiring comments on early versions of this paper.

\section{References}

1. Christopher Anderson, Paola Giannini, and Sophia Drossopoulou. Towards type inference for JavaScript. In Proc. 19th European Conference on Object-Oriented Programming, ECOOP '05, volume 3586 of LNCS. Springer-Verlag, July 2005.

2. Shay Artzi, Adam Kiezun, Julian Dolby, Frank Tip, Danny Dig, Amit M. Paradkar, and Michael D. Ernst. Finding bugs in dynamic web applications. In Proc. International Symposium on Software Testing and Analysis, ISSTA '08. ACM, July 2008.

3. Darren C. Atkinson and William G. Griswold. Implementation techniques for efficient data-flow analysis of large programs. In Proc. International Conference on Software Maintenance, ICSM '01, pages 52-61, November 2001.

4. Gogul Balakrishnan and Thomas W. Reps. Recency-abstraction for heap-allocated storage. In Proc. 13th International Static Analysis Symposium, SAS '06, volume 4134 of LNCS. Springer-Verlag, August 2006.

5. David R. Chase, Mark Wegman, and F. Kenneth Zadeck. Analysis of pointers and structures. In Proc. ACM SIGPLAN Conference on Programming Language Design and Implementation, PLDI'90, June 1990.

6. Patrick Cousot and Radhia Cousot. Abstract interpretation: a unified lattice model for static analysis of programs by construction or approximation of fixpoints. In Proc. 4th ACM SIGPLAN-SIGACT Symposium on Principles of Programming Languages, POPL 'rry, pages 238-252, 1977.

7. Christian Fecht and Helmut Seidl. Propagating differences: An efficient new fixpoint algorithm for distributive constraint systems. In Programming Languages and Systems, Proc. 7th European Symposium on Programming, ESOP '98, volume 1381 of LNCS. Springer-Verlag, March/April 1998.

8. Michael Furr, Jong hoon (David) An, Jeffrey S. Foster, and Michael W. Hicks. Static type inference for Ruby. In Proc. ACM Symposium on Applied Computing, SAC '09, 2009.

9. Arjun Guha, Shriram Krishnamurthi, and Trevor Jim. Using static analysis for Ajax intrusion detection. In Proc. 18th International Conference on World Wide Web, $W W W$ '09, 2009.

10. Phillip Heidegger and Peter Thiemann. Recency types for analyzing scripting languages. In Proc. 24th European Conference on Object-Oriented Programming, ECOOP '10, LNCS. Springer-Verlag, June 2010.

11. Michael Hind. Pointer analysis: haven't we solved this problem yet? In Proc. ACM SIGPLAN-SIGSOFT Workshop on Program Analysis For Software Tools and Engineering, PASTE '01, pages 54-61, June 2001. 
12. Michael Hind, Michael G. Burke, Paul R. Carini, and Jong-Deok Choi. Interprocedural pointer alias analysis. ACM Transactions on Programming Languages and Systems, 21(4):848-894, 1999.

13. Susan Horwitz, Alan Demers, and Tim Teitebaum. An efficient general iterative algorithm for dataflow analysis. Acta Informatica, 24(6):679-694, 1987.

14. Susan Horwitz, Thomas Reps, and Mooly Sagiv. Demand interprocedural dataflow analysis. In Proc. 3rd ACM SIGSOFT Symposium on Foundations of Software Engineering, FSE '95, October 1995.

15. Simon Holm Jensen, Anders Møller, and Peter Thiemann. Type analysis for JavaScript. In Proc. 16th International Static Analysis Symposium, SAS '09, volume 5673 of $L N C S$. Springer-Verlag, August 2009.

16. Neil D. Jones and Steven S. Muchnick. A flexible approach to interprocedural data flow analysis and programs with recursive data structures. In Proc. 9th ACM Symposium on Principles of Programming Languages, POPL '82, January 1982.

17. John B. Kam and Jeffrey D. Ullman. Global data flow analysis and iterative algorithms. Journal of the ACM, 23(1):158-171, 1976.

18. John B. Kam and Jeffrey D. Ullman. Monotone data flow analysis frameworks. Acta Informatica, 7:305-317, 1977. Springer-Verlag.

19. Gary A. Kildall. A unified approach to global program optimization. In Proc. 1st ACM Symposium on Principles of Programming Languages, POPL 'r3, October 1973.

20. Barbara Liskov and Stephen N. Zilles. Programming with abstract data types. ACM SIGPLAN Notices, 9(4):50-59, 1974.

21. Peter W. O'Hearn, John C. Reynolds, and Hongseok Yang. Local reasoning about programs that alter data structures. In Proc. 15th International Workshop on Computer Science Logic, CSL '01, September 2001.

22. Simon L. Peyton Jones. The Implementation of Functional Programming Languages. Prentice Hall, 1987.

23. Thomas Reps, Susan Horwitz, and Mooly Sagiv. Precise interprocedural dataflow analysis via graph reachability. In Proc. 22th ACM SIGPLAN-SIGACT Symposium on Principles of Programming Languages, POPL '95, pages 49-61, January 1995.

24. Barbara G. Ryder, William Landi, Phil Stocks, Sean Zhang, and Rita Altucher. A schema for interprocedural modification side-effect analysis with pointer aliasing. ACM Transactions on Programming Languages and Systems, 23(2):105-186, 2001.

25. Shmuel Sagiv, Thomas W. Reps, and Susan Horwitz. Precise interprocedural dataflow analysis with applications to constant propagation. Theoretical Computer Science, 167(1\&2):131-170, 1996.

26. Micha Sharir and Amir Pnueli. Two approaches to interprocedural dataflow analysis. In Program Flow Analysis: Theory and Applications, pages 189-233. PrenticeHall, 1981.

27. Peter Thiemann. Towards a type system for analyzing JavaScript programs. In Proc. Programming Languages and Systems, 14th European Symposium on Programming, ESOP '05, April 2005.

28. Teck Bok Tok, Samuel Z. Guyer, and Calvin Lin. Efficient flow-sensitive interprocedural data-flow analysis in the presence of pointers. In Proc. 15th International Conference on Compiler Construction, CC '06, pages 17-31, March 2006.

29. Yichen Xie and Alex Aiken. Static detection of security vulnerabilities in scripting languages. In Proc. 15th USENIX Security Symposium, August 2006. 


\section{A Theoretical Properties}

The lazy propagation analysis framework is supposed to improve on the results of the basic framework in several respects. First, the modifications should not affect termination. Second, analysis results with lazy propagation should always be at least as precise as in the basic framework, meaning that the extensions introduce no spurious results. Third, the extensions should be sound in the sense that the analysis result is still a fixpoint of the transfer functions, which has to be adjusted because of the introduction of unknown field values, and that the transfer functions remain meaningful with respect to the language semantics. In the following, we state these properties more precisely and study them in some detail.

\section{A.1 Termination}

As observed in Section 3, the AnalysisLattice modifications do not preserve monotonicity of the transfer functions. Nevertheless, it is easy to see that the worklist algorithm (Figure 1) always terminates.

Proposition 1. The worklist algorithm always terminates in the lazy propagation framework.

Proof. Each AnalysisLattice operation terminates. The only nontrivial case is recover: Its first phase clearly terminates as only a finite set of nodes is considered, and the second phase terminates because AnalysisLattice has finite height.

Every iteration of the worklist algorithm removes a location from the worklist, and transfer functions only add new locations to the worklist when the lattice element is modified. As every such modification makes the lattice element larger and the lattice has finite height, termination is ensured.

The number of iterations required to reach the fixpoint may differ due to the modifications. First, as mentioned in Section 2.3, we have left the worklist processing order unspecified and that order may be affected by the modifications. Second, as described in Section 3, the operation funexit' improves precision with respect to the original funexit operation by avoiding certain interprocedurally invalid paths. Depending on the particular analysis instance, this improved precision may result in an increase or in a decrease of the number of iterations required to compute the fixpoint. In practice, we observe an overall decrease on each of our benchmark programs, as shown in Section 4.

The cost of performing a recover operation is proportional to the number of times it applies $\sqcup$. In the basic framework, the same amount of work is done, although "eagerly" within propagate operations. Hence, recovery does not impair the amortized analysis complexity. 




Fig. 8. Mapping between lattices in the extended and the basic framework.

\section{A.2 Precision}

For clarity, the text in this subsection marks all elements and lattices from the lazy propagation framework with primes ' whereas entities from the basic framework remain unadorned. Let $a_{0} \in$ AnalysisLattice be a solution of an analysis instance $\mathcal{A}$ in the basic framework, and let $a^{\prime} \in$ AnalysisLattice' $^{\prime}$ be an intermediate step arising during the fixpoint iteration in the extended framework for $\mathcal{A}$. The goal is to show that $a^{\prime}$ is always smaller than $a_{0}$ in the lattice ordering, but this ordering cannot be directly established because the two lattices are different. Hence, we first need a function $\alpha$ that maps values of the extended analysis to values of the basic analysis. Figure 8 contains the definition of this function on the various lattices. It is easily seen to be bottom-preserving, monotone, and distributive.

The property that no spurious results arise with lazy propagation can now be stated as an invariant of the while loop in the worklist algorithm from Figure 1.

Proposition 2. Let $\mathcal{A}$ be an analysis instance, $a_{0} \in$ AnalysisLattice be the solution of $\mathcal{A}$ in the basic framework, and $a^{\prime} \in$ AnalysisLattice' be the analysis lattice element on an entry to the while loop in the worklist algorithm (Figure 1) applied to $\mathcal{A}$ with the lazy propagation framework. Then $a^{\prime}$ and $a_{0}$ are $\alpha$-related, i.e., $\alpha\left(a^{\prime}\right) \sqsubseteq a_{0}$.

Proof. On first entry to the loop, $a^{\prime}=\perp_{\text {AnalysisLattice }}$. As $\alpha$ is bottom-preserving, $\alpha\left(a^{\prime}\right) \sqsubseteq a_{0}$. To establish the invariant, we assume that $\alpha\left(a^{\prime}\right) \sqsubseteq a_{0}$, let $t=$ $T\left(c_{0}, n_{0}\right)$, for some $\left(c_{0}, n_{0}\right) \in C \times N$, and show that $\alpha\left(t\left(a^{\prime}\right)\right) \sqsubseteq a_{0}$.

As part of the computation of $t\left(a^{\prime}\right)$, the transfer function $t$ may invoke the ADT operations on $a^{\prime}$, and we need to (1) check the effect of each operation on $a^{\prime}$ and prove that the $\alpha$ relation still holds. Additionally, since the output of one operation may be used as input to another and we may assume that the arguments of each invocation of an operation in a transfer function are computed by monotone functions, we are also obliged to (2) check that $\alpha$-related arguments to the operations yield $\alpha$-related results. In the following, we let $\left(m_{0}, g_{0}\right)=a_{0}$ and $\left(m^{\prime}, g^{\prime}\right)=a^{\prime}$ and prove the properties (1) and (2) for each operation in turn.

Case getcallgraph ${ }^{\prime}$. The invocation of $a^{\prime}$.getcallgraph'() does not affect $a^{\prime}$. The result is a subset of $a_{0}$.getcallgraph () because $\alpha\left(a^{\prime}\right) \sqsubseteq a_{0}$.

Case getstate. This operation does not modify $a^{\prime}$. For the result, we have $\alpha\left(a^{\prime} . \operatorname{getstate}(c, n)\right) \sqsubseteq a_{0} . \operatorname{getstate}(c, n)$. 
Case getfield ${ }^{\prime}$. Consider the invocation of $a^{\prime}$.getfield $(c, n, \ell, p)$. If $m^{\prime}(c, n)=$ none, then $a^{\prime}$ is not changed and the result is $\perp$ which preserves the invariant. Let now $m^{\prime}(c, n) \neq$ none and $v=a^{\prime}$.getfield $(c, n, \ell, p)$. If $v \neq$ unknown, then $a^{\prime}$ is not changed and $\alpha(v) \sqsubseteq a_{0}$.getfield $(c, n, \ell, p)$. If $v=$ unknown, then we need to consider the changes effected by recover where we also relate the result to the expected one.

Case propagate . Consider the invocation of $a^{\prime}$.propagate $\left(c, n, s^{\prime}\right)$ from a transfer function $t=T\left(c_{0}, n_{0}\right)$, where $\left(c_{0}, n_{0}\right)$ is a predecessor of $(c, n)$. As $a_{0}$ is a solution, it holds that $t\left(a_{0}\right) \sqsubseteq a_{0}$ and that consequently $a_{0}$.propagate $(c, n, s)$ leaves $a_{0}$ unchanged, where $\alpha\left(s^{\prime}\right) \sqsubseteq s$ as both states are computed by the same monotone function from $\alpha$-related arguments.

If $u^{\prime}=m^{\prime}(c, n)$ is none, then $m^{\prime}(c, n)$ is effectively updated to $s^{\prime}$. Now, $\alpha\left(m^{\prime}(c, n)\right)=\alpha\left(s^{\prime}\right) \sqsubseteq s \sqsubseteq m(c, n)$ with the last equation holding because $a_{0}$.propagate leaves $a_{0}$ unchanged.

Otherwise, parts of $u^{\prime}$ may need to be recovered which (assumedly) does not violate the invariant. We then have that $\alpha\left(m^{\prime}(c, n)\right) \sqsubseteq m(c, n)$ before the invocation of propagate and $\alpha\left(m^{\prime}(c, n) \sqcup s^{\prime}\right)=\alpha\left(m^{\prime}(c, n)\right) \sqcup \alpha\left(s^{\prime}\right) \sqsubseteq m(c, n) \sqcup s \sqsubseteq$ $m(c, n)$ afterwards.

This operation returns no result, so the $\alpha$-relation trivially holds.

Case recover. Consider the invocation of $a^{\prime}$.recover $(c, n, \ell, p)$. The first node added to the graph $G$ is $(c, f u n(n))$.

For this return value, it holds that $\alpha\left(v^{\prime}\right) \sqsubseteq m(c$, entry $(f u n(n)))(\ell)(p)$ by assumption. By similar reasoning as in subcase B below, it must be that

$$
m(c, \operatorname{entry}(f u n(n)))(\ell)(p) \sqsubseteq m(c, n)(\ell)(p)=a_{0} . \operatorname{getfield}(c, n, \ell, p) .
$$

Hence, $\alpha\left(v^{\prime}\right) \sqsubseteq a_{0}$. getfield $(c, n, \ell, p)$ as required.

Once the graph $G$ has been constructed, the recovery algorithm first examines the roots $\left(c^{\prime}, f^{\prime}\right)$ of $G$ and modifies their states in $a^{\prime}$. Let $\left(c^{\prime}, f^{\prime}\right)$ be such a root, $u^{\prime}=m^{\prime}\left(c^{\prime}\right.$,entry $\left.\left(f^{\prime}\right)\right)$, and let $\left(c_{1}, n_{1}\right)$ be such that $u_{g}^{\prime}=g^{\prime}\left(c_{1}, n_{1}, c^{\prime}, f^{\prime}\right) \neq$ none. Let further $u_{c}^{\prime}=m^{\prime}\left(c_{1}, n_{1}\right)$ and $u_{1}^{\prime}=m^{\prime}\left(c_{1}\right.$,entry $\left.\left(\operatorname{fun}\left(n_{1}\right)\right)\right)$.

As $\left(c^{\prime}, f^{\prime}\right)$ is reachable there must have been a prior step in the fixpoint iteration where some transfer function $t^{\prime}=T\left(c_{1}, n_{1}\right)$ invokes funentry $y^{\prime}$ Inside of this $t^{\prime}$ there must be a monotone function invoke which commutes with $\alpha$ and which constructs the State argument to funentry' such that $u_{g}^{\prime}=\operatorname{invoke}\left(u_{c}^{\prime}\right)$. This same function is also used in the verification that $a_{0}$ is a solution. In this verification, suppose that the State argument is $s=i n v o k e\left(u_{c}\right)$ where $u_{c}=$ $m_{0}\left(c_{1}, n_{1}\right)$. Let further $u=m_{0}\left(c^{\prime}, \operatorname{entry}\left(f^{\prime}\right)\right)$ and $u_{1}=m_{0}\left(c_{1}, \operatorname{entry}\left(f u n\left(n_{1}\right)\right)\right)$.

Subcase A. Let us first assume that $u_{g}^{\prime}(\ell)(p) \neq$ unknown. By our assumptions, it holds that $\alpha\left(u^{\prime}\right) \sqsubseteq u$ and $\alpha\left(u_{c}^{\prime}\right) \sqsubseteq u_{c}$. Because $u_{g}^{\prime}=\operatorname{invoke}\left(u_{c}^{\prime}\right)$ and $s=$ invoke $\left(u_{c}\right)$ and invoke commutes with $\alpha$, it also holds that $\alpha\left(u_{g}^{\prime}\right) \sqsubseteq s$.

Now, let $u_{g}^{\prime \ell p}$ be bottom except at $\ell . p$ where it is equal to $u_{g}^{\prime}(\ell)(p)$. With this setting, we can reason that

$$
\begin{aligned}
\alpha\left(u^{\prime} \sqcup u_{g}^{\prime \ell p}\right) & \sqsubseteq \alpha\left(u^{\prime} \sqcup u_{g}^{\prime}\right)=\alpha\left(u^{\prime} \sqcup \operatorname{invoke}\left(u_{c}^{\prime}\right)\right) \\
& =\alpha\left(u^{\prime}\right) \sqcup \alpha\left(\operatorname{invoke}\left(u_{c}^{\prime}\right)\right) \sqsubseteq u \sqcup \operatorname{invoke}\left(u_{c}\right)=u
\end{aligned}
$$


where the last equality is due to the propagate operation in the standard funentry operation.

Subcase B. For the second case, assume that $u_{g}^{\prime}(\ell)(p)=$ unknown but $u_{1}^{\prime}(\ell)(p) \neq$ unknown. As the algorithm propagates the latter value, we need to prove that it would not change if it were propagated to $u_{c}^{\prime}$. In fact, to establish the invariant it is sufficient to show that $u_{1}(\ell)(p) \sqsubseteq u_{c}(\ell)(p)$ in the basic analysis.

Suppose for a contradiction that $u_{1}(\ell)(p) \nsubseteq u_{c}(\ell)(p)$. Then there must be some $n_{x}$ on a path between $n_{e}=\operatorname{entry}\left(\operatorname{fun}\left(n_{1}\right)\right)$ and $n_{1}$ where each node between $n_{e}$ and $n_{x}$ satisfies $u_{1}(\ell)(p) \sqsubseteq m_{0}\left(c_{1}, n_{e}\right)(\ell)(p)$ but $u_{1}(\ell)(p) \nsubseteq m_{0}\left(c_{1}, n_{x}\right)(\ell)(p)$. Let $n_{x}^{\prime}$ be the predecessor of $n_{x}$ on this path. Clearly, $T\left(c_{1}, n_{x}^{\prime}\right)$ changes the $\ell . p$ field by invoking propagate $\left(c_{1}, n_{x}^{\prime}, s_{x}\right)$ for some $s_{x}=\operatorname{action}\left(m_{0}\left(c_{1}, n_{x}^{\prime}\right)\right)$ with $s_{x}(\ell)(p) \sqsupset \perp$.

As the same transfer function must have been called in the extended framework (otherwise the function call at $n_{1}$ would not be reachable), there must have been an invocation of propagate $\left(c_{1}, n_{x}^{\prime}, s_{x}^{\prime}\right)$ for some $s_{x}^{\prime}$ with $\alpha\left(s_{x}^{\prime}\right) \sqsubseteq s_{x}$ and $s_{x}^{\prime}(\ell)(p) \sqsupset \perp$ (because $T$ never processes unknown). But such an invocation contradicts $u_{g}^{\prime}(\ell)(p)=$ unknown, so no such node $n_{x}$ exists.

Hence, $\alpha\left(u_{1}^{\prime}(\ell)(p)\right) \sqsubseteq u_{1}(\ell)(p) \sqsubseteq u_{c}(\ell)(p)$ so that

$$
\begin{aligned}
\alpha\left(u^{\prime} \sqcup u_{1}^{\prime}\right)(\ell)(p) & \sqsubseteq \alpha\left(u^{\prime} \sqcup u_{1}^{\prime}\right)(\ell)(p) \\
& =\alpha\left(u^{\prime}\right)(\ell)(p) \sqcup \alpha\left(u_{1}^{\prime}\right)(\ell)(p) \\
& \sqsubseteq u(\ell)(p) \sqcup u_{1}(\ell)(p) \\
& \sqsubseteq u(\ell)(p) \sqcup u_{c}(\ell)(p) \\
& \sqsubseteq u(\ell)(p) \sqcup \text { invoke }\left(u_{c}\right)(\ell)(p) \\
& =u(\ell)(p)
\end{aligned}
$$

Thus, recovery at the roots does not violate the desired invariant. The final propagation does not do so either. It propagates state from the function entry node of the caller to the function entry node of the callee under the assumption that the corresponding component on the call edge is unknown. This assumption holds by construction of $G$. With the same argumentation as in the previous case, the state of the $\ell . p$ field cannot change between the entry to the caller and the actual call, so the invariant holds after each iteration of the loop and thus for the fixpoint as well.

The return value is extracted from $m^{\prime}(c, e n t r y(f u n(n)))(\ell)(p)$ which $\alpha$ approximates the value $a_{0}$. getfield $(c, n, \ell, p)$ as explained in the beginning of this case.

Case funentry'. An invocation of $a^{\prime}$. funentry $\left(c_{1}, n_{1}, c_{2}, f_{2}, s^{\prime}\right)$ first adds $s^{\prime}$ to the call edge, which is correct because the corresponding call to funentry $\left(c_{1}, n_{1}\right.$, $\left.c_{2}, f_{2}, s\right)$ in the basic framework adds the tuple $\left(c_{1}, n_{1}, c_{2}, f_{2}\right)$ to the basic call graph.

Next it computes a projection $s^{\prime \prime}$ of $s^{\prime}$, for which clearly $s^{\prime \prime} \sqsubseteq s^{\prime}$ and hence $\alpha\left(s^{\prime \prime}\right) \sqsubseteq s$ holds. With this precondition, the call to propagate preserves the invariant. 
If the final call to funexit' does not happen, then there is no further change to $a^{\prime}$. Otherwise, the invariant holds by assumption on funexit.

This operation returns no result, so again the $\alpha$-relation trivially holds.

Case funexit'. Each invocation $a^{\prime}$.funexit $\left(c_{1}, n_{1}, c_{2}, f_{2}, s^{\prime}\right)$ happens with a state argument computed from the exit node of function $f_{2}$, such as, $n_{2}=$ $\operatorname{exit}\left(f_{2}\right)$, so that $s^{\prime}=f_{\text {exit }}\left(m^{\prime}\left(c_{2}, n_{2}\right)\right)$. Hence, the analogous call in the verification of the basic framework uses $s=f_{\text {exit }}\left(m\left(c_{2}, n_{2}\right)\right)$, so that $\alpha\left(s^{\prime}\right) \sqsubseteq s$ holds, as usual.

Let furthermore $u_{g}^{\prime}=g^{\prime}\left(c_{1}, n_{1}, c_{2}, f_{2}\right)$ be the corresponding call edge and $u_{g}$ the state parameter of the corresponding funentry call in the basic framework.

Let $L P=\left\{(\ell, p) \mid s^{\prime}(\ell, p)=\right.$ unknown $\}$. By similar reasoning as in the case for recover, for each $(\ell, p) \in L P$, it holds that $u_{g}(\ell)(p) \sqsubseteq m\left(c_{2}, n_{2}\right)(\ell)(p)$, that is, this state component is preserved from the invocation to the end of the function.

For the state $s^{\prime \prime}$ computed in funexit' we must argue that $\alpha\left(s^{\prime \prime}\right) \sqsubseteq s$ which is not obvious. For $(\ell, p) \notin L P$, it holds that $\alpha\left(s^{\prime \prime}(\ell)(p)\right)=\alpha\left(s^{\prime}(\ell)(p)\right) \sqsubseteq s(\ell)(p)$ by assumption $\alpha\left(s^{\prime}\right) \sqsubseteq s$. For $(\ell, p) \in L P$, it holds that $\alpha\left(s^{\prime \prime}(\ell)(p)\right)=\alpha\left(u_{g}^{\prime}(\ell)(p)\right) \sqsubseteq$ $u_{g}(\ell)(p) \sqsubseteq m\left(c_{2}, n_{2}\right)(\ell)(p)=s(\ell)(p)$.

Hence, the final call to propagate ${ }^{\prime}$ happens with $\alpha$-related arguments and does not destroy the invariant.

This operation returns no result, so again the $\alpha$-relation trivially holds.

\section{A.3 Soundness}

The changes made to the AnalysisLattice operations indirectly modify the transfer functions, so it is also important that these remain sound with respect to the semantics of the program. To state this more precisely, let $\llbracket Q \rrbracket$ be a collecting semantics of a program $Q$ (in the abstract interpretation sense [6]) such that $\beta_{\llbracket Q \rrbracket}$ is an abstraction of $\llbracket Q \rrbracket$ in the domain AnalysisLattice from Section 2 expressed via the operations getfield and getcallgraph. We say that $a \in$ AnalysisLattice (using either the basic framework or lazy propagation) over-approximates $\beta_{\llbracket Q \rrbracket}$ if



We conjecture that lazy propagation is then sound in the following sense:

Assume that $a_{0} \in$ AnalysisLattice is the solution in the basic analysis framework of an analysis instance $\mathcal{A}$ for a program $Q$ and that $a_{0}$ over-approximates $\beta_{\llbracket Q \rrbracket}$. If $a_{0}^{\prime}$ is the solution of $\mathcal{A}$ in the lazy propagation framework then $a_{0}^{\prime}$ also overapproximates $\beta_{\llbracket Q \rrbracket}$.

Without giving a full proof, we mention some key aspects of the reasoning. Most importantly, lazy propagation gives a safe approximation compared to the maybe-modified technique briefly mentioned in Section 2.5, and that technique is clearly sound relative to the basic framework.

The worklist algorithm for the basic framework produces a solution to the analysis in the sense defined in Section 2.3. A requirement for this to hold is that 
every AnalysisLattice ADT operation that modifies an abstract state at some location also adds that location to the worklist. This requirement is also fulfilled with lazy propagation - except for a subtlety in the recover operation: It modifies states that belong to function entry locations without adding these to the worklist. This means that such values that have been recovered at the function entry locations may not be propagated. However, recall that transfer functions can only read object field values via the getfield' operation. Assume that $\operatorname{getfield}^{\prime}(c, n, \ell, p)$ is invoked and the field $\ell . p$ is unknown at the location $(c, n)$. In that case, getfield ${ }^{\prime}$ will call recover, and in the situation where the proper value $v$ has already been recovered at the function entry location $(c, \operatorname{entry}(\operatorname{fun}(n)))$ the value $v$ is returned by getfield'. This means that the transfer function will behave in the same way as if $v$ had been propagated from the function entry location. A similar situation occurs if the recovery has taken place not at the same function but at an earlier location in the call graph. Thus, the fact that recover modifies abstract states without adding their locations to the worklist does not affect correctness of the analysis result. 